ARTICLE

Received 31 Jul 2012 | Accepted 19 Oct 2012 | Published 20 Nov 2012

DOI: $10.1038 /$ ncomms2213

\title{
Hopping transport and the Hall effect near the insulator-metal transition in electrochemically gated poly(3-hexylthiophene) transistors
}

\author{
Shun Wang ${ }^{1}$, Mingjing $\mathrm{Ha}^{1}$, Michael Manno ${ }^{1}, \mathrm{C}$. Daniel Frisbie ${ }^{1} \&$ C. Leighton ${ }^{1}$
}

Despite 35 years of investigation, much remains to be understood regarding charge transport in semiconducting polymers, including the ultimate limits on their conductivity. Recently developed ion gel gating techniques provide a unique opportunity to study such issues at very high charge carrier density. Here we have probed the benchmark polymer semiconductor poly(3-hexylthiophene) at electrochemically induced three-dimensional hole densities approaching $10^{21} \mathrm{~cm}^{-3}$ (up to 0.2 holes per monomer). Analysis of the hopping conduction reveals a remarkable phenomenon where wavefunction delocalization and Coulomb gap collapse are disrupted by doping-induced disorder, suppressing the insulator-metal transition, even at these extreme charge densities. Nevertheless, at the highest dopings, we observe, for the first time in a polymer transistor, a clear Hall effect with the expected field, temperature and gate voltage dependencies. The data indicate that at such mobilities $\left(\sim 0.8 \mathrm{~cm}^{2} \mathrm{~V}^{-1} \mathrm{~s}^{-1}\right.$ ), despite the extensive disorder, these polymers lie close to a regime of truly diffusive band-like transport.

\footnotetext{
${ }^{1}$ Department of Chemical Engineering and Materials Science, University of Minnesota, Minneapolis, Minnesota 55455, USA. Correspondence and requests for materials should be addressed to C.L. (email: leighton@umn.edu).
} 
D espite a 35-year effort, semiconducting polymers continue to provide serious challenges to our understanding of charge transport in solids ${ }^{1-4}$. Elusive issues include the non-trivial influence of the multiple sources of disorder (for example, semi-crystallinity, paracrystallinity and nanoscale inhomogeneity ${ }^{1-7}$ ), the elucidation of the important hopping mechanisms ${ }^{1-4,7-10}$, the effects of charge carrier-lattice interactions $^{3,4}$ and the identification of the ultimate limits on conductivity and mobility ${ }^{1-6,11}$. The latter is particularly important as, in addition to being of considerable fundamental interest the upper limits on conductivity and mobility also limit device performance (for example, transistor output current and operating frequency). In this context it is important to note that in the vast majority of cases these semiconducting polymers populate the insulating side of the Mott-Anderson insulatormetal transition, where transport occurs by thermally assisted hopping between localized electronic states (for example, refs 1-4 and 7-9). In fact, it is only in a limited number of cases (for example, free-standing thick films of semiconducting polymers such as polyaniline (for example, refs 11 and 12) or polypyrrole (for example, ref. 13)) that chemical doping can delocalize carrier wavefunctions to the point of inducing an insulator-metal transition to a state with finite conductivity as $T \rightarrow 0$, or even entering the weakly localized regime beyond it $^{12,13}$. It is currently unclear whether a metallic state is possible only in certain semiconducting polymers, or whether efficacy of chemical doping, or minimization of disorder, limit delocalization of the charge carriers.

In addition, and in contrast to lower disorder organic conductors such as small molecule systems, the anticipated crossover from non-adiabatic hopping to diffusive band transport with increasing carrier density has not yet been observed in semiconducting polymers. It has been argued that such a crossover is expected when the mobility reaches a quasi-universal value of $\sim 1 \mathrm{~cm}^{2} \mathrm{~V}^{-1} \mathrm{~s}^{-1}$ (ref. 4). In one of the most important recent advances in organic electronics, this value has now been surpassed in electrostatically gated surface layers of single-crystal small molecule semiconductors such as rubrene (for example, refs 14 and 15). Electrostatic gating in the field-effect transistor geometry is ideal for such studies, as it enables wide-range tuning of the carrier density in a single sample ${ }^{16}$. A clear crossover to band transport has indeed been detected in such devices, via observation of a phonon-limited temperature dependence of the mobility, a robust Hall effect, and equivalence of the Hall mobility and the mobility extracted from gate voltagedependent measurements ${ }^{14,15}$. Semiconducting polymers, perhaps unsurprisingly given their lower crystallinity, low bandwidth and greater disorder, lag somewhat behind. Although the mobilities of benchmark semiconducting polymers such as poly (3-hexylthiophene; $\mathrm{P} 3 \mathrm{HT}$ ) are now at, or are approaching, the $1 \mathrm{~cm}^{2} \mathrm{~V}^{-1} \mathrm{~s}^{-1}$ threshold in thin film transistors ${ }^{17,18}$, no phononlimited mobility has been observed and no Hall effect has been reported.

In this work we exploit the recent developments with ionic liquid/gel gating techniques in thin film transistors to study electrochemically doped P3HT at three-dimensional (3D) hole densities up to $10^{21} \mathrm{~cm}^{-3}$, that is, 0.2 holes per monomer. An insulator-metal transition does not take place even at these extreme densities, an effect that can be traced back via detailed analysis of the variable-range hopping (VRH) conduction to doping-induced disorder, preventing carrier wavefunction delocalization and Coulomb gap collapse. The results thus point to a serious potential limitation of electrochemical doping in such systems. Nevertheless, at the highest dopings and temperatures, we observe for the first time in a semiconducting polymer thin film transistor, a robust Hall signal with expected field, temperature and gate voltage dependencies. The data suggest that at these mobilities $\left(\sim 0.8 \mathrm{~cm}^{2} \mathrm{~V}^{-1} \mathrm{~s}-1\right)$, despite the extensive disorder, such solution-processed films lie close to a regime of truly diffusive band-like transport.

\section{Results}

Electrochemical thin film transistors. A schematic of the device studied (see Methods section for synthesis details) is shown in Fig. 1a. An optical micrograph and further characterization details are provided in Supplementary Fig. S1. In essence, the device is a four-terminal bottom-contacted van der Pauw geometry P3HT thin film transistor, gated electrochemically using an ion gel based on an ionic liquid (1-ethyl-3-methylimidazolium bis(trifluoro-methylsulfonyl) imide ((EMIM)(TFSI))) and a triblock copolymer (poly(styrene- $b$-methylmethacrylate- $b$-styrene; PS-PMMA-PS) $)^{19-21}$. Such ionic liquids and gels have been used recently to electrostatically induce two-dimensional charge densities $\sim 10^{14} \mathrm{~cm}^{-2}$ in a variety of materials (for example, refs 22-25). In the case of semi-crystalline semiconducting polymers such as P3HT, the open structure results in deep penetration of the dopant ions, providing uniform $3 \mathrm{D}$ electrochemical doping (Fig. 1b) ${ }^{21,26}$. That the P3HT thickness employed here $(60 \mathrm{~nm})$ can be safely assumed to be in the $3 \mathrm{D}$ doping limit is well established ${ }^{21,26}$. Although quantification of P3HT crystallinity remains a challenge, it is generally regarded to be quite low ${ }^{6}$, and heavy electrochemical doping is expected to further reduce it. We thus view the system as predominantly amorphous. However, the P3HT used here is of a high-enough molecular weight $\left(M_{\mathrm{n}}=23 \mathrm{kDa}\right.$, see Methods) to lie on the saturation region of the mobility versus the $M_{\mathrm{n}}$ curve $\mathrm{e}^{27}$, approaching $1 \mathrm{~cm}^{2} \mathrm{~V}^{-1} \mathrm{~s}^{-1}$ in annealed devices.

Critical to the success of this study, which involves cryogenic transport measurements over a wide carrier density range on a single device, was the development of device designs and fabrication methods, resulting in robust, repeatable transport properties over long time periods and multiple thermal cycles. This is illustrated in Fig. 1c, which shows $280 \mathrm{~K}$ transfer curves (that is, source-drain current $\left(I_{\mathrm{d}}\right)$ versus gate voltage $\left(V_{\mathrm{G}}\right)$ plots) for a single device over a 5-week period and $>30$ thermal cycles. The device has on/off ratios approaching $10^{6}$, threshold voltages around $-0.5 \mathrm{~V}$ and modest $V_{\mathrm{G}}$ hysteresis. Bias-stress effects are negligible, enabling drift-free isothermal magnetic field sweeps for facile measurement of magnetoresistance and the Hall effect. Aside from the decrease in off current, which we ascribe to a gradual decrease in oxygen content, no significant change in performance with time is observed.

As a simple measure of the total injected charge density, $Q$, the gate current during charging was integrated over long times (for example, see inset in Fig. 1d at $V_{\mathrm{G}}=-0.30,-0.65$ and $-1.00 \mathrm{~V})$. The main panel of Fig. $1 \mathrm{~d}$ shows the result of repeating this procedure from -0.3 to $-1.0 \mathrm{~V}$ at $280 \mathrm{~K}$, the $-1.0 \mathrm{~V}$ upper limit being set by the irreversible decrease in conductivity that occurs beyond this voltage with this particular ionic liquid. Note that this irreversible conductivity decrease at high $\left|V_{\mathrm{G}}\right|$ is not universal. In transistors based on some organic conductor/ionic liquid (or gel) combinations, reversible conductivity decreases are observed at high $\left|V_{\mathrm{G}}\right|$ (for example, ref. 26). The highest hole densities $(p)$ estimated are around $8 \times 10^{20} \mathrm{~cm}^{-3}$, corresponding (right axis) to 0.2 holes per thiophene ring. In the main set of experiments presented here, the devices are first electrochemically gated to the desired $V_{\mathrm{G}}$, the gate current integrated and then cooled below the freezing temperature of the ion gel $(\sim 220 \mathrm{~K})$ to fix $p$. All subsequent measurements are made in the Ohmic response regime as shown in Supplementary Fig. S2 and discussed in the Methods section. 
a
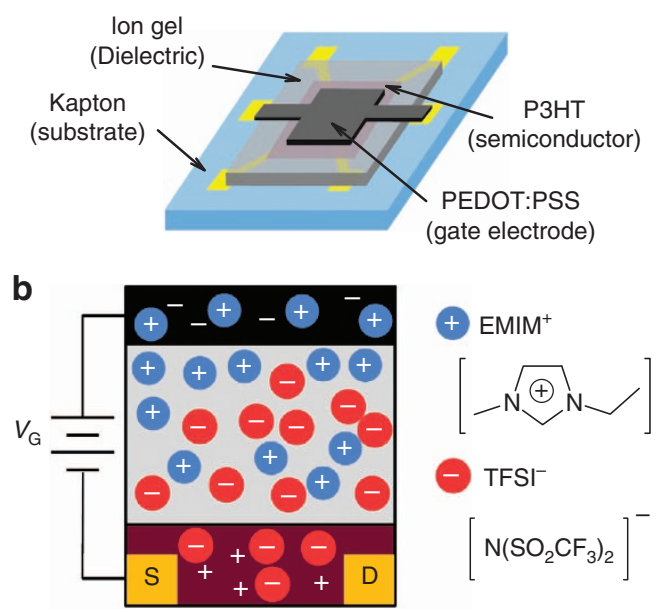
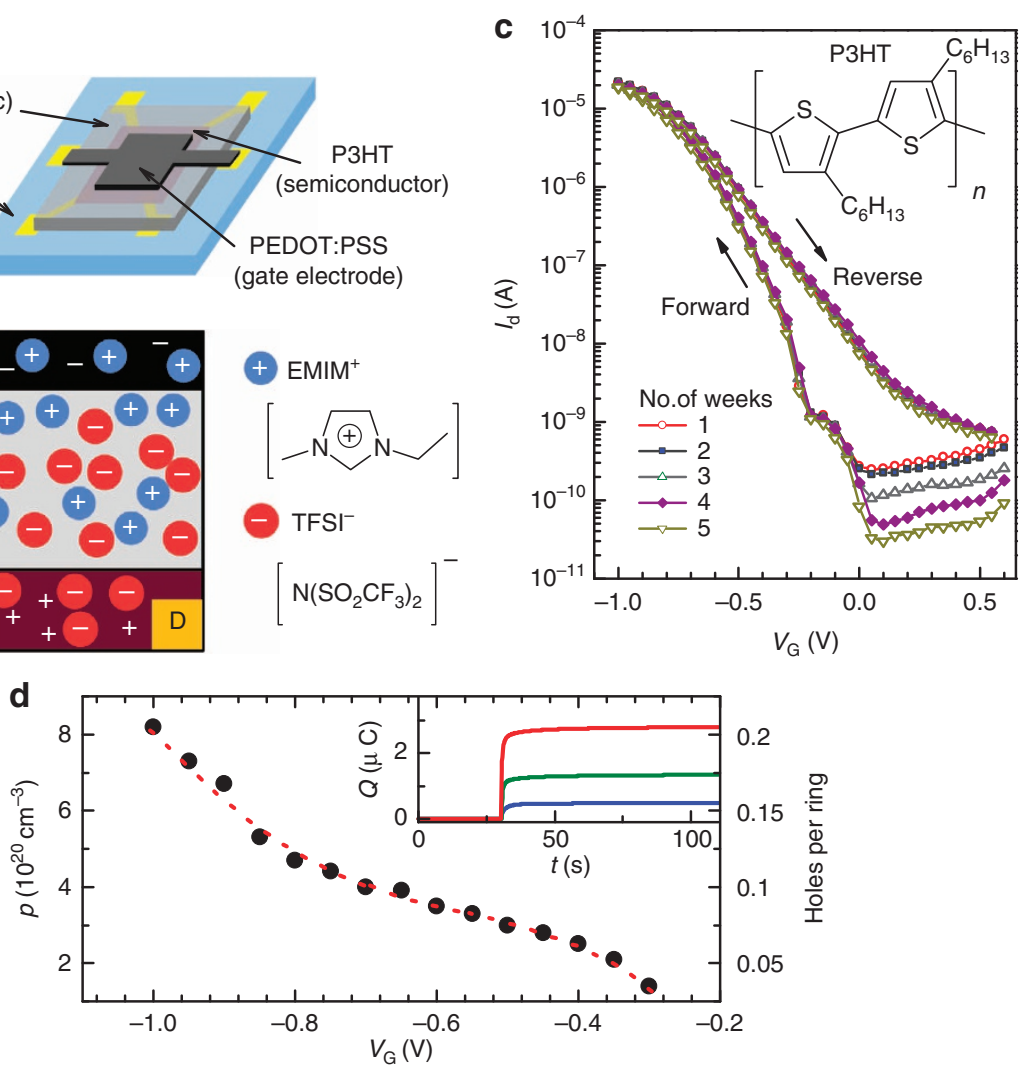

Figure 1 | Schematics and $\mathbf{I - V}$ characteristics of electrochemically gated P3HT transistors. (a) Schematic of the van der Pauw geometry device structure (channel size is $500 \mu \mathrm{m} \times 500 \mu \mathrm{m}$ ). An optical image of an actual device is shown in Supplementary Fig. S1. (b) Schematic of charge injection into the polymer semiconductor. The top layer (black) is the gate electrode, the middle layer (grey) is the ion gel and the bottom layer (maroon) is the polymer semiconductor. Simple ' + ' and ' - ' symbols are used for electronic charge carriers, and blue and red symbols are used for ions. The chemical structures of $\mathrm{EMIM}^{+}$and TFSI- ${ }^{-}$are shown on the right. (c) Drain current $\left(I_{\mathrm{d}}\right)$ versus gate voltage $\left(V_{\mathrm{G}}\right)$ at $280 \mathrm{~K}$ measured 1 week apart for 5 consecutive weeks. $V_{\mathrm{G}}$ was swept at $50 \mathrm{mVs}^{-1}$. The inset shows the chemical structure of P3HT. (d) Gate voltage $\left(V_{\mathrm{G}}\right)$ dependence of the hole density $(p)$. The right axis shows the conversion to holes per thiophene ring. The inset shows accumulated charge ( $Q$, integrated from the gate current) versus time. The top (red), middle (green) and bottom (blue) curves are for $-1.00,-0.65$ and $-0.30 \mathrm{~V}$, respectively.

Hopping transport. By varying $V_{\mathrm{G}}$ from -0.3 to $-1.0 \mathrm{~V}$ in $0.05 \mathrm{~V}$ increments, we measured the temperature dependence of the resistivity, $\rho$, at 15 distinct $p$ values between $\sim 10^{20}$ and $10^{21} \mathrm{~cm}^{-3}$ in a single electrochemical device. The data are plotted as $\rho$ (log scale) versus $T$ in Supplementary Fig. S2(c), and as $\rho$ (log scale) versus $T^{-1 / 2}$ and $T^{-1 / 4}$ in Fig. $2 \mathrm{a}, \mathrm{b}$, down to $1.5 \mathrm{~K}$. Although the $250 \mathrm{~K}$ resistivity can be decreased by well over three orders of magnitude to values as low as $12 \mathrm{~m} \Omega \mathrm{cm}$ (a conductivity of $\left.83 \mathrm{scm}^{-1}\right), \mathrm{d} \rho / \mathrm{d} T$ remains negative even at $p=8 \times 10^{21} \mathrm{~cm}^{-3}$. Indeed, the conductivity does not extrapolate to finite values as $T \rightarrow$ 0 , indicating that an insulator-metal transition is absent. Rather, we shall show that Fig. $2 \mathrm{a}-\mathrm{c}$ demonstrate conclusively that the mechanism of conduction is actually VRH. As shown in Fig. 2a, $\log (\rho)$ is linear in $T^{-1 / 2}$ over the whole accessible $T$ range for $p<6 \times 10^{20} \mathrm{~cm}^{-3}$. For $p>6 \times 10^{20} \mathrm{~cm}^{-3}, \log (\rho)$ is linear in $T^{-1 / 2}$ only at low $T$ (Fig. 2a), being instead linear in $T^{-1 / 4}$ at high $T$ (Fig. 2b). Verification of these deduced $T$ exponents, via an unbiased quantitative analysis, was achieved via the logarithmic derivative method, where $w=-\mathrm{d}(\ln \rho) / \mathrm{d}(\ln T)$ is plotted as a function of $\ln T$ to linearize $\rho=\rho_{0} \exp \left(T_{0} / T\right)^{x}$, yielding $-x$ directly from the slope. As can be seen from Fig. $2 c$, this process confirms $x \sim 0.5$ over the whole $T$ range for low $p\left(<6 \times 10^{20} \mathrm{~cm}^{-3}\right)$, and a T-dependent crossover from $x \sim 0.5$ to $x \sim 0.25$ at high $p(>6 \times$ $10^{20} \mathrm{~cm}^{-3}$ ). Critically, and as returned to below, at the highest $p$ values only, the data of Fig. $2 c$ also suggest the existence of a crossover to a third regime (arrow in Fig. 2c) at high $T$.
Given the 3D nature of this system, the $T^{-1 / 4}$ behaviour at relatively high $T$ can be readily identified as Mott $\mathrm{VRH}$, where $\rho=\rho_{\text {Mott }} \exp \left(T_{\text {Mott }} / T\right)^{1 / 4}$, with $\rho_{\text {Mott }}$ being the $T \rightarrow \infty$ value of $\rho(T)$ and $T_{\text {Mott }}$ being a characteristic temperature ${ }^{28}$. In general, $T_{\text {Mott }}=18 / k_{\mathrm{B}} L_{\mathrm{C}}^{3} N\left(E_{\mathrm{F}}\right)$, where $L_{\mathrm{C}}$ is the localization length (that is, the decay length of the exponentially localized wavefunctions) and $N\left(E_{\mathrm{F}}\right)$ is the density of states at the Fermi level, $E_{\mathrm{F}}$ (ref. 28). The $T^{-1 / 2}$ behaviour, however, could be interpreted in a number of ways, particularly conventional Efros-Shklovskii (ES) $\mathrm{VRH}^{29}$ or inter-cluster hopping ${ }^{30}$. In conventional ES VRH, Coulomb interaction effects lead to a soft-gapped density of states around $E_{\mathrm{F}}$ giving $\rho=\rho_{\mathrm{ES}} \exp \left(T_{\mathrm{ES}} / T\right)^{1 / 2}$, where $T_{\mathrm{ES}}=2.8 e^{2} / \kappa k_{\mathrm{B}} L_{\mathrm{C}}$, and $\kappa$ is the dielectric constant ${ }^{29}$. A similar $T$ dependence can, however, also be obtained from thermally assisted tunnelling between nanoscopic conductive regions in a more insulating matrix, due to the Coulomb penalty associated with single carrier charging ${ }^{30}$. Such models have been adapted to semiconducting polymers ${ }^{8}$ and claims of observation of this mechanism have been made ${ }^{9}$. We thus performed additional analyses and measurements to clarify the origin of the $T^{-1 / 2}$ behaviour.

The first of these analyses is displayed in the inset to Fig. 3a, which compares the observed temperature of crossover between $T^{-1 / 2}$ and $T^{-1 / 4}$ regimes, and the predicted crossover temperature, assuming ES VRH. The latter occurs at $16 T_{\mathrm{ES}}^{2} / T_{\mathrm{Mott}}$ (refs 9,31) and, as demonstrated by the straight line fit with slope 1 , the agreement with the experiment is very good, strongly 

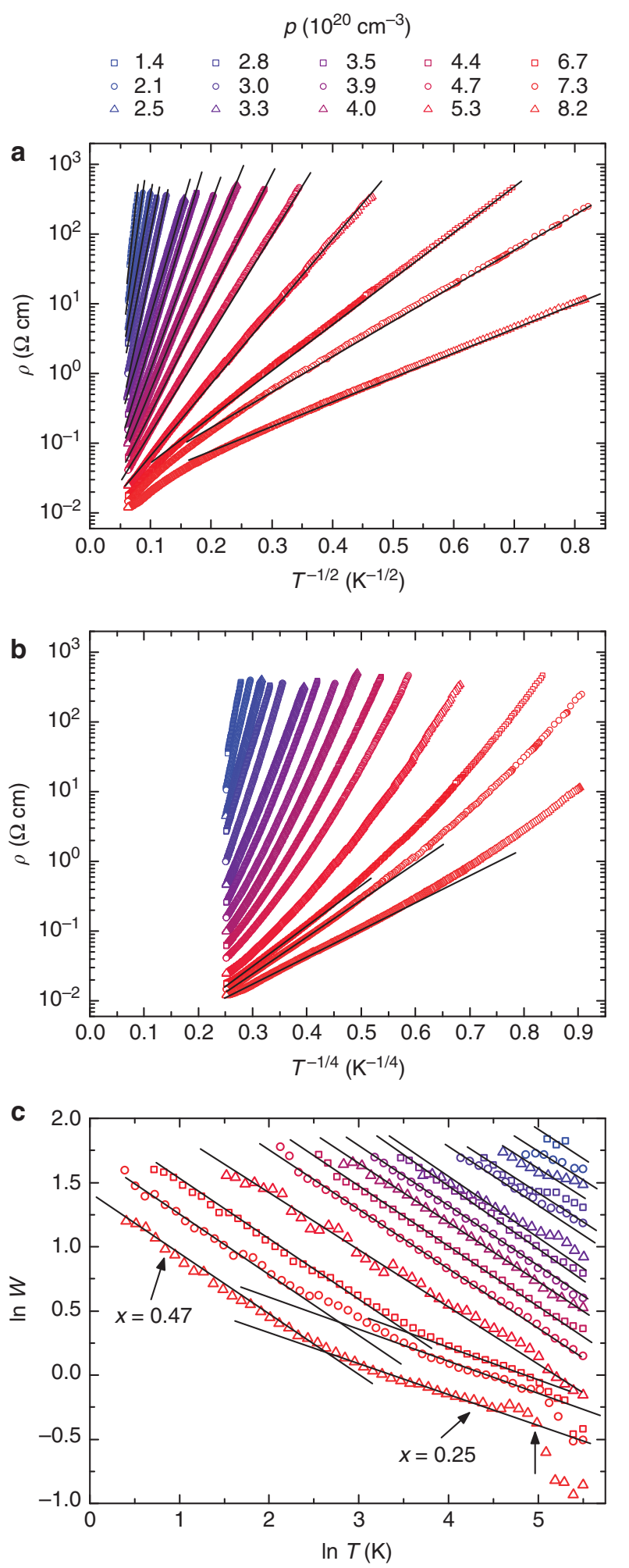

Figure 2 | Temperature dependence of the zero magnetic field resistivity ( $\rho$ ). (a) $\ln \rho$ versus $T^{-1 / 2}$ for 15 distinct gate-induced carrier densities (in units of $10^{20} \mathrm{~cm}^{-3}$ ), in a single device. The solid lines are straight line guides to the eye. (b) In $\rho$ versus $T^{-1 / 4}$ for the same 15 carrier densities. The solid lines are straight line guides to the eye. (c) In $W$ versus In $T$ plot for the same 15 carrier densities, where $W$, the logarithmic derivative, is defined as $-d(\ln \rho) / d(\ln T)$. The solid lines correspond to $x \sim-0.5$ and $x=-0.25$. The vertical arrow indicates the departure from hopping behaviour. The symbol key shown at the top of panel a applies to panels a-c.
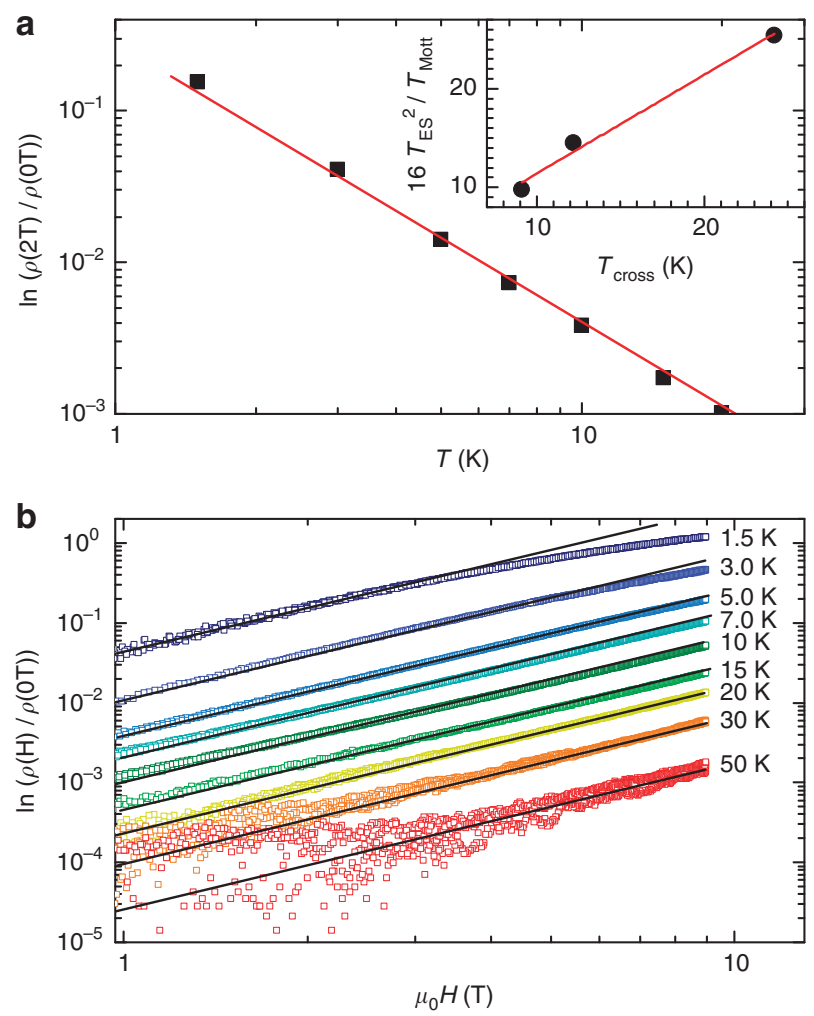

Figure 3 | Magnetoresistance. (a) Log-log plot of $\ln \left(\rho\left(\mu_{0} H=2 T\right) / \rho(0)\right)$ versus $T$ at $p=8.2 \times 10^{21} \mathrm{~cm}^{-3}$. $H$ is the applied magnetic field. The solid line has a slope of -1.8 . The inset shows the predicted crossover temperature from ES to Mott VRH (that is, $16 T_{\mathrm{ES}}^{2} / T_{\text {Mott }}$ ) versus the observed crossover temperature, $T_{\text {cross }}$. The solid line is a linear fit with a slope of 1.0. (b) Log-log plot of $\ln (\rho(H) / \rho(0))$ versus $\mu_{0} H$ for temperatures from 1.5 to $50 \mathrm{~K}$ at $p=8.2 \times 10^{21} \mathrm{~cm}^{-3}$. The solid lines have slopes of 1.9 .

supporting interpretation as conventional ES VRH. Further evidence is provided by magnetoresistance measurements with magnetic field $(H)$ perpendicular to the device plane, where large positive magnetoresistance is expected due to field-induced suppression of wavefunction overlap. In the weak field limit, ES VRH is expected to result in $\ln (\rho(H) / \rho(0))=t(e / c h)^{2} H^{2} L_{C}^{4}(T /$ $\left.T_{0}\right)^{-1.5}$, with $t=0.0015$ (ref. 29). Representative data at $p=8.2 \times 10^{21} \mathrm{~cm}^{-3}$ are presented as a function of $T$ and $H$ in Fig. 3a,b, respectively. Figure $3 \mathrm{~b}$ confirms the existence of large positive magnetoresistance increasing rapidly with decreasing $T$, with a $\ln (\rho(H) / \rho(0)) \propto H^{1.9}$ dependence, in good agreement with theory. Figure $3 \mathrm{a}$ reveals $\ln (\rho(2 \mathrm{~T}) / \rho(0)) \propto T^{-1.8}$, again in reasonable agreement with theory for ES VRH. Indeed, with the exception of the small negative deviations from the predicted $H$ dependence at the lowest $T$ (which are quite common but do not correspond to simple estimates for the crossover from the weak- to high-field limits), we conclude that the ES VRH accounts quantitatively for all aspects of our low $T$ data. We interpret the apparent adherence to conventional models of $\mathrm{VRH}$, with no modifications for charge carrier-lattice or polaronic effects, as evidence that in the majority of the parameter space studied here we are in a regime of strongly non-adiabatic transport with hopping energies significantly smaller than polaron transport or formation energies. As discussed in more detail below, we believe that the deviations from hopping behaviour at high $T$ and $p$ in Fig. $2 c$ signal a crossover to a more adiabatic regime where polaronic effects may be more important. 

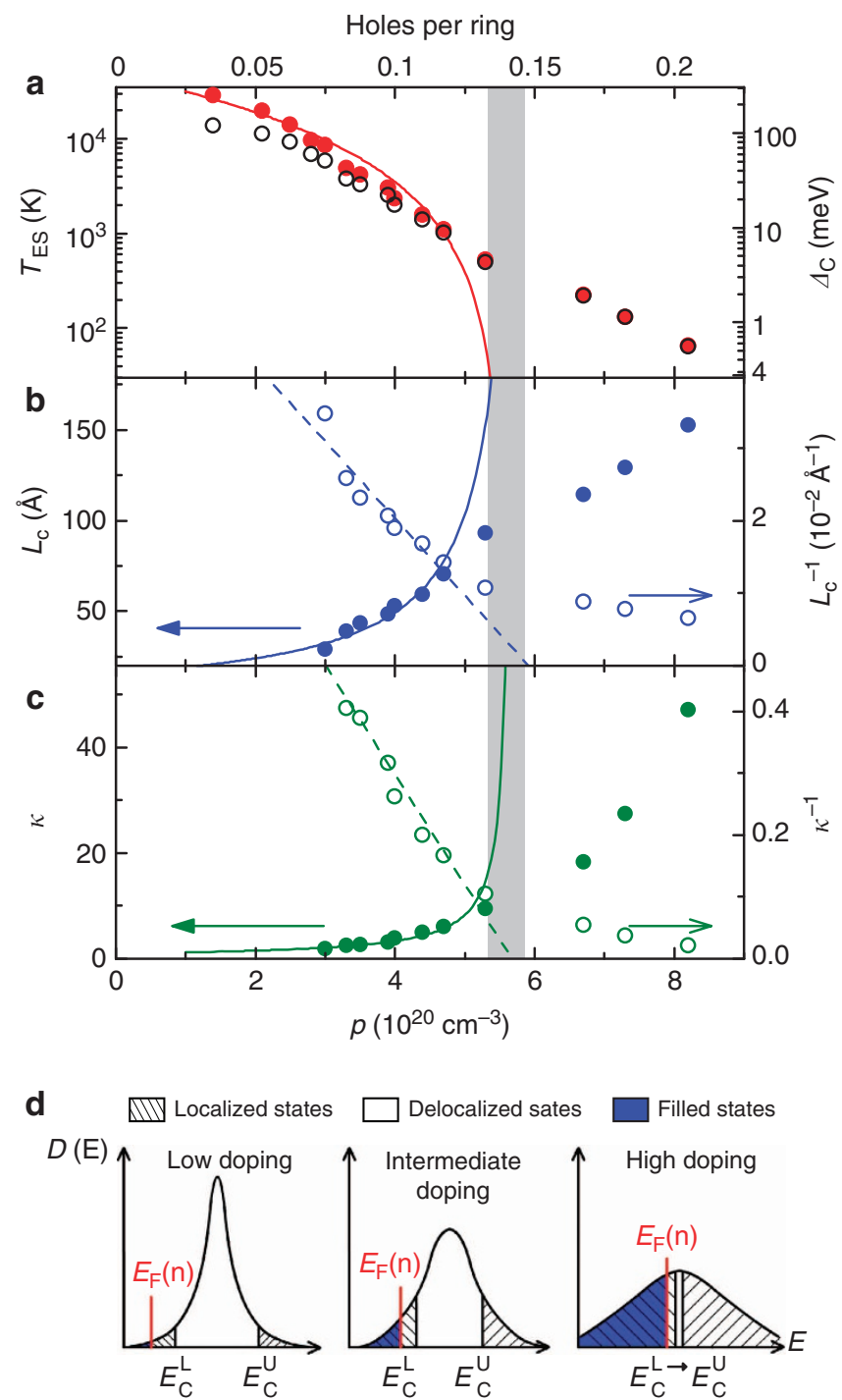

Figure 4 | The approach to the insulator-metal transition. Panels a-c show the doping dependence of the characteristic temperature of the ES hopping $\left(T_{\mathrm{ES}}\right)$, the localization length $\left(L_{C}\right)$ and the dielectric constant $(\varepsilon)$. These quantities are plotted as solid symbols, corresponding to the left axes. The open symbols (corresponding to the right axes) show, respectively, the conversion to the Coulomb gap $\left(\Delta_{\mathrm{C}}\right)(\mathbf{a})$, and the inverse localization length (b) and dielectric constant (c). The top axis shows the conversion to holes per thiophene ring. The solid (dashed) lines in a-c are fits to $T_{\mathrm{ES}} L_{C}\left(L_{C}^{-1}\right)$ and $\varepsilon\left(\varepsilon^{-1}\right)$ for $p<5 \times 10^{20} \mathrm{~cm}^{-3}$. The fitting equations (parameters) are $T_{\mathrm{ES}}=T_{0}\left(1-p / p_{\mathrm{C}}\right)^{2}\left(T_{0}=47,000 \mathrm{~K}\right.$ and $\left.p_{\mathrm{c}}=5.5 \times 10^{20} \mathrm{~cm}^{-3}\right), L_{\mathrm{C}}=L_{\mathrm{CO}}\left(1-p / p_{\mathrm{c}}\right)^{-1}\left(L_{\mathrm{CO}}=16 \AA\right.$ and $\left.p_{\mathrm{c}}=5.9 \times 10^{20} \mathrm{~cm}^{-3}\right)$ and $\varepsilon=\varepsilon_{0}\left(1-p / p_{\mathrm{c}}\right)^{-1}\left(\varepsilon_{0}=1.3\right.$ and $\left.p_{c}=5.7 \times 10^{20} \mathrm{~cm}^{-3}\right)$. The grey shaded region represents the vicinity of the hypothetical insulator-metal transition in the absence of dopinginduced disorder. (d) Schematic of the evolution of the HOMO (highest occupied molecular orbital) density of states with electrochemical doping. $E_{\mathrm{F}}(p)$ is the doping-dependent Fermi level, and $E_{C} \mathrm{~L}_{\mathrm{U}} \mathrm{U}$ are the mobility edges in the upper and lower region of the HOMO.

It is natural to question why the crossover from Mott to ES VRH occurs at such high $T$. In inorganic semiconductors, for instance, this crossover is observed below $1 \mathrm{~K}$ (for example, ref. 32), when thermal fluctuations are lowered to the point that the Coulomb gap is no longer smeared. Insight into this is provided by Fig. $4 \mathrm{a}$, which plots $T_{\mathrm{ES}}(p)$, the right axis showing the conversion (see Supplementary Methods) to $\Delta_{\mathrm{C}}$, the Coulomb gap width. The values (up to $100 \mathrm{meV}$ at the lowest $p$ ) are large cf. conventional inorganic semiconductors, an observation that was previously used to argue for some form of inter-cluster hopping over $\mathrm{ES} \mathrm{VRH}^{9}$. As discussed above, we believe the evidence for ES VRH is overwhelming here; we rather interpret the high $T_{\mathrm{ES}}$ and $\Delta_{\mathrm{C}}$ in terms of the low $\kappa$ values in semiconducting polymers via $\Delta_{\mathrm{C}}=e^{3} N\left(E_{\mathrm{F}}\right)^{1 / 2} / \kappa^{3 / 2}$. There are, in fact, obvious parallels with the relative importance of excitonic effects in organic and inorganic semiconductors.

Approach to the insulator-metal transition. In Fig. $4 a-c$, we plot the $p$ dependence of the other parameters extracted from the VRH analysis of $\rho(T, H)$. Figure $4 \mathrm{~b}$ shows $L_{C}(p)$ extracted from magnetoresistance data, using the formula provided above. Note that very similar $L_{C}$ values are obtained regardless of whether we fit the $T$ dependence at fixed $H$ (Fig. 3a), or the $H$ dependence at fixed $T$ (Fig. $3 b)$. Figure $4 c$ then plots $\kappa(p)$ deduced from $T_{\mathrm{ES}}(p)$ and $L_{\mathrm{C}}(p)$ via $T_{\mathrm{ES}}=2.8 e^{2} / \kappa k_{\mathrm{B}} L_{\mathrm{C}}$. Although the qualitative behaviour is as expected ( $T_{\mathrm{ES}}$ drops with $p$ due to increases in $L_{\mathrm{C}}$ and $\kappa)$, quantitatively these results lie far from simple expectations. Specifically, the expected divergences in $L_{\mathrm{C}}$ and $\kappa$ with increasing $p$ as the insulator-metal transition is approached, should follow the scaling theory of electron localization, that is, $L_{\mathrm{C}}=L_{\mathrm{C} 0}\left(1-p / p_{\mathrm{c}}\right)^{-v}$ and $\kappa=\kappa_{0}\left(1-p / p_{\mathrm{c}}\right)^{-\alpha}$, where $p_{\mathrm{c}}$ is the critical carrier density for the insulator-metal transition, and $L_{\mathrm{C} 0}$ and $\kappa_{0}$ are the undoped values of $L_{\mathrm{C}}$ and $\kappa^{28}$. This leads to the collapse of the Coulomb gap according to $T_{\mathrm{ES}}=T_{\mathrm{ES} 0}$ $\left(1-p / p_{c}\right)^{v+\alpha}$. Although controversy remains over the precise values of the exponents, $v$ is often found to be unity, whereas $\alpha$ is often 1 or 2 (refs 28 and 29). It is abundantly clear from Fig. $4 a-c$ that these scaling forms are violated in our case. $L_{C}$ and $\kappa$ are anomalously weakly $p$-dependent, no divergence taking place, no collapse in $\Delta_{\mathrm{C}}$ occurring and, thus, no insulator-metal transition being induced. As shown in Supplementary Fig. S3, these $V_{\mathrm{G}}$ dependencies of $T_{\mathrm{ES}}$ and $L_{\mathrm{C}}$, and indeed all other transport parameters, are robust, being observed in multiple devices. Further insight is obtained by fitting only the low $p$ region $\left(p<5 \times 10^{20} \mathrm{~cm}^{-3}\right)$ of $T_{\mathrm{ES}}(p), L_{\mathrm{C}}(p)$ and $\kappa(p)$ to the expected scaling forms. Purely for the purpose of demonstration, we fix $v$ and $\alpha$ at 1 , at which point we find that reasonable fits to all three quantities can be obtained with a single $p_{\mathrm{c}}$ value around $5.7 \times 10^{20} \mathrm{~cm}^{-3}$. This is illustrated by the right axes of Fig. $4 \mathrm{~b}, \mathrm{c}$, which plot $L_{\mathrm{C}}^{-1}(p)$ and $\kappa^{-1}(p)$, yielding reasonable linear fits for $p<5 \times 10^{20} \mathrm{~cm}^{-3}$. In essence, at low doping $L_{C}$ and $\kappa$ increase in a manner consistent with the scaling theory, and a hypothetical $p_{\mathrm{c}} \sim 5.7 \times 10^{20} \mathrm{~cm}^{-3}$ ( 0.14 dopant ions per monomer). Before this doping value is reached, however, adherence to scaling is lost, $L_{\mathrm{C}}$ and $\kappa$ become weakly $p$-dependent, no divergences occur and, thus, the insulator-metal transition does not take place. Note that the low $p$ fits also yield perfectly reasonable values for $L_{\mathrm{C} 0}$ and $\kappa_{0}$; we find $L_{\mathrm{C} 0} \sim 15 \AA$, corresponding to $\sim 3$ thiophene rings, and $\kappa_{0} \sim 1.5$, reasonably close to the accepted value $^{33}$

We propose that the key concept to understand the anomalous doping dependence of the parameters shown in Fig. $4 \mathrm{a}-\mathrm{c}$ is electrochemical doping-induced disorder. The $p$ value at which adherence to the expected scaling forms is lost corresponds to $>0.1$ dopant ions per monomer. We hypothesize that at these extreme electrochemical doping levels, the forced insertion of dopant ions into the P3HT leads to substantial increases in disorder, likely to the point of additional loss in crystallinity. This competes with the increase in hole density, preventing divergence of $L_{\mathrm{C}}$ and $\kappa$, and thus collapse of $\Delta_{\mathrm{C}}$. In essence, $p_{\mathrm{c}}$ becomes doping-dependent. To further illustrate this concept, Fig. $4 \mathrm{~d}$ provides sketches of the available density of states, $D(E)$, in the 
narrow valence band of P3HT at low $\left(<5 \times 10^{20} \mathrm{~cm}^{-3}\right)$, intermediate $\left(\sim 5 \times 10^{20} \mathrm{~cm}^{-3}\right)$ and high $p\left(>5 \times 10^{20} \mathrm{~cm}^{-3}\right)$. We consider the situation where at low $p$ some delocalized states exist near the middle of the band, separated from the exponentially localized ones in the band tails by the lower and upper mobility edges, $E_{\mathrm{C}}^{\mathrm{L}}$ and $E_{\mathrm{C}}^{\mathrm{U}}$. As $p$ is increased, $E_{\mathrm{F}}(p)$ rises, $L_{\mathrm{C}}$ and $\kappa$ begin to diverge in accordance with the scaling theory, $\Delta_{\mathrm{C}}$ falls and an insulator-metal transition is anticipated as $E_{\mathrm{F}}(p) \rightarrow$ $E_{\mathrm{C}}^{\mathrm{L}}$. However, the increasing disorder associated with the high levels of electrochemical doping required to reach this point leads to additional Anderson localization and an increase in $E_{\mathrm{C}}^{\mathrm{L}}$ with $p$, thus suppressing the insulator-metal transition. As is hopefully clear from the illustration of the high $p$ regime in Fig. 4 d, it is thus unclear whether an insulator-metal transition is possible with this type of doping, that is, whether $E_{\mathrm{F}}$ can be increased sufficiently before the region of delocalized states between $E_{\mathrm{C}}^{\mathrm{L}}$ and $E_{\mathrm{C}}^{\mathrm{U}}$ vanishes. This may in fact be directly relevant to the puzzling recent observations of reversible decreases in conductance at high $\left|V_{\mathrm{G}}\right|$ in similar devices ${ }^{26}$.

Hall effect. Although the insulator-metal transition is not reached in our devices, the distinct departure from hopping behaviour in $\rho(T)$ above $\sim 150 \mathrm{~K}$ at the highest $p$ values (Fig. $2 \mathrm{c}$ ) raises the possibility of a departure from the non-adiabatic hopping regime, potentially to a regime of diffusive transport. We thus searched for a Hall effect in the high $p$, high $T$ region. Although the Hall effect has been reported sporadically in bulk semiconducting polymer samples on the metallic side of the insulator-metal transition ${ }^{11,34}$, its detection in thin film transistors and on the insulating side of the transition has traditionally been thwarted by low mobilities and high contact resistances. In our case, the mobility values are comparable to the best reported in $\mathrm{P} 3 \mathrm{HT}$ thin film transistors (approaching $1 \mathrm{~cm}^{2} \mathrm{~V}^{-1} \mathrm{~s}^{-1}$ ), and contact resistances are minimized by electrochemical doping ${ }^{35}$. As demonstrated by the representative $190 \mathrm{~K}$ data shown in the inset to Fig. 5 (more data are provided in Supplementary Figs S4 and S5), a robust Hall effect is indeed observed at the three highest $\left|V_{\mathrm{G}}\right|$ values. The Hall resistivity $\left(\rho_{\text {Hall }}\right)$ has the expected response to sign reversal of the current and magnetic field, is linear in $H$, and yields a positive Hall coefficient $\left(R_{\mathrm{H}}\right)$, consistent with hole transport (see Supplementary Fig. S5). It must be emphasized that $R_{\mathrm{H}}$ increases with increasing $\left|V_{\mathrm{G}}\right|$. We believe this signals the emergence from the hopping regime (where the Hall effect is unmeasurably small) and the onset of a crossover towards diffusive transport. The extent to which this crossover is complete is clarified by the data in the main panel of Fig. 5, where the $p$ dependence of the mobility, $\mu(p)=1 / p e \rho(p)$, is plotted using $p$ values extracted both from our standard integration of the gate current $\left(\mu_{\text {Gate }}\right)$ and from the Hall effect $\left(\mu_{\text {Hall }}\right)$. For this purpose, the Hall effect is assumed to be dominated by a single carrier type; the observed linearity with magnetic field is important in this regard. A rapid, three order of magnitude increase in $\mu_{\text {Gate }}$ is observed on increasing $p$ from 1 to $5 \times 10^{20} \mathrm{~cm}^{-3}$, followed by a much slower increase at the highest $p$. The $\mu_{\mathrm{Hall}}$ on the other hand is first detected around $6.5 \times 10^{20} \mathrm{~cm}^{-3}$ $\left(0.05 \mathrm{~cm}^{2} \mathrm{~V}^{-1} \mathrm{~s}^{-1}\right)$, increasing relatively quickly at higher $p$. We find $\mu_{\text {Hall }}(p)<\mu_{\text {Gate }}(p)$ at all $p$, although the two values converge asymptotically in the high $p$ limit. This again reflects the emergence from the non-adiabatic hopping regime (where $R_{\mathrm{H}}$ is very small) and the crossover towards diffusive transport where $\mu_{\text {Hall }}(p) \sim \mu_{\text {Gate }}(p)$ is expected. That this crossover is not complete is further clarified by $\mu_{\mathrm{Hall}}(T)$ (see Supplementary Fig. S4, at $V_{\mathrm{G}}=-1 \mathrm{~V}$ ), which reveals a monotonic two-fold decrease in $\mu_{\text {Hall }}$ on cooling from 190 to $30 \mathrm{~K}$, demonstrating that $\mu_{\mathrm{Hall}}(T)$ is

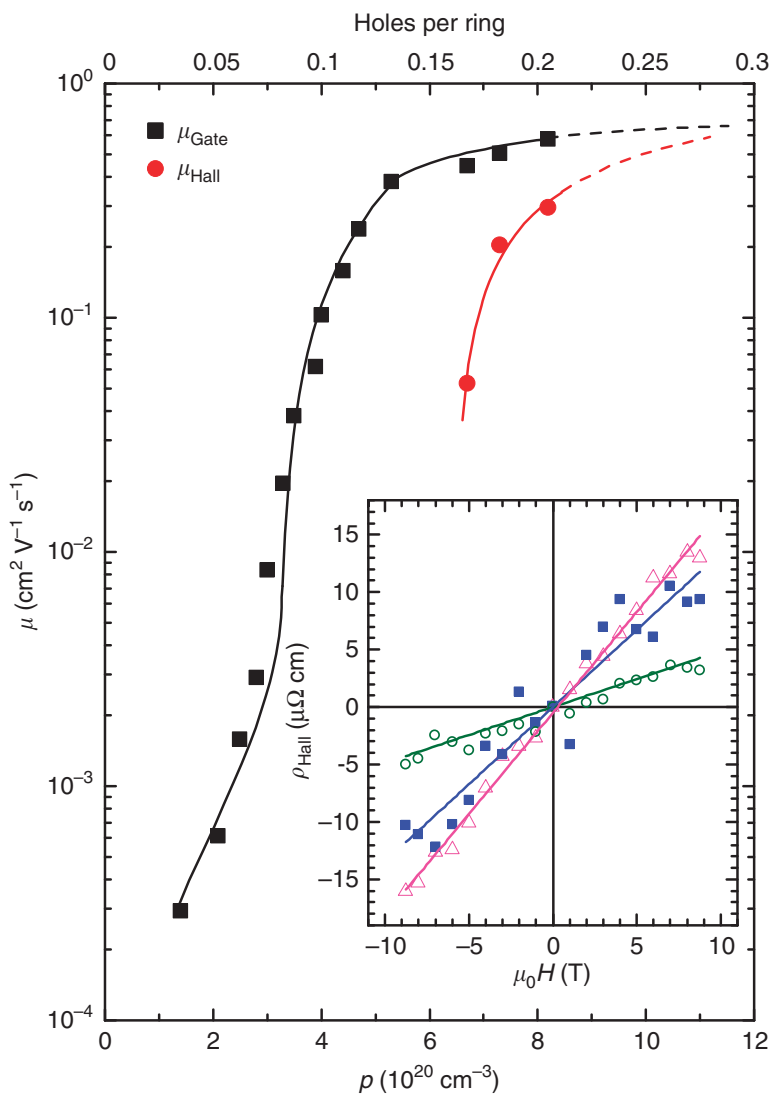

Figure 5 | Observation of the Hall effect. Hole density dependence of the $190 \mathrm{~K}$ mobility $(\mu)$ extracted from the integrated charge and measured via the Hall effect. Inset: Hall resistivity $\left(\rho_{\text {Hall }}\right)$ versus $\mu_{0} H$ for three different gate voltages at $190 \mathrm{~K} ;-1.00 \mathrm{~V}$ (red triangles), $-0.95 \mathrm{~V}$ (blue squares) and $-0.90 \mathrm{~V}$ (green circles). The zero field Hall resistivity has been subtracted from the data. The solid lines are linear fits.

not yet phonon-limited. We, in fact, observe weak carrier freeze-out, an Arrhenius plot of $p(T)$ yielding activation energies as low as $2.5 \mathrm{meV}$ (Supplementary Fig. S4).

\section{Discussion}

We believe that the picture that emerges from this work is quite clear. At mobilities approaching $1 \mathrm{~cm}^{2} \mathrm{~V}^{-1} \mathrm{~s}^{-1}$ conductive polymer thin film transistors can apparently be doped to the verge of the crossover from a strongly non-adiabatic VRH regime to an adiabatic, diffusive, band-like transport regime, where a Hall signal can be detected for the first time. This occurs despite the identification of a potential limitation of electrochemical doping, where doping-induced disorder limits carrier wavefunction delocalization. The results indicate that truly diffusive band-like transport is likely within reach in low-temperature solution-processed plastic transistors, an advance that would open up myriad opportunities in organic electronics.

\section{Methods}

Device fabrication. The raw materials consisted of (a) P3HT (Rieke Metals, measured $M_{\mathrm{n}}$ of $23 \mathrm{kDa}$ ); (b) an ion gel ink composed of a mixture of $1.5 \mathrm{wt} \%$ poly(styrene- $b$-methylmethacrylate- $b$-styrene (PS-PMMA-PS) triblock copolymer $\left(M_{\mathrm{n}}=13-65-13 \mathrm{kDa}\right.$, polydispersity 1.3$), 8.5 \mathrm{wt} \%$ 1-ethyl-3-methylimidazolium bis(trifluoro-methylsulfonyl) imide ((EMIM)(TFSI)) ionic liquid (Merck), and $90 \mathrm{wt} \%$ ethyl acetate; and (c) PEDOT:PSS ink ( $\mathrm{PH} 500,1-1.4 \mathrm{wt} \%$ polymer in $\mathrm{H}_{2} \mathrm{O}$, from H.C. Stark), diluted with $10 \mathrm{vol} \%$ ethylene glycol to enhance conductivity. To fabricate 4 -probe $500 \times 500 \mu \mathrm{m}$ devices, $\mathrm{Cr}(2.5 \mathrm{~nm}) / \mathrm{Au}(25 \mathrm{~nm})$ bottom electrodes were first photolithographically patterned on Kapton substrates (Dupont) of thickness $50 \mu \mathrm{m}$. P3HT was then spin-coated from a chloroform 
solution $\left(6 \mathrm{mg} \mathrm{ml}^{-1}\right)$ to a thickness of $60 \mathrm{~nm}$. The root-mean-square roughness of the bare Kapton substrates and the P3HT film were found to be $\sim 1 \mathrm{~nm}$ over a $5 \times 5-\mu \mathrm{m}$ area by atomic force microscopy (see Supplementary Fig. S1). The P3HT layer was then manually patterned using a chloroform-soaked swab. The ion gel and gate electrodes ( 5 and $1 \mu \mathrm{m}$ thick, respectively) were subsequently printed using a commercial aerosol-jet system (Optomec, Inc., see ref. 18 for details), and the device was baked in a $\mathrm{N}_{2}$-filled glove box for $3 \mathrm{~h}$ at $110^{\circ} \mathrm{C}$.

Measurement details. Electronic transport measurements were performed in $\mathrm{He}$ vapour in a commercial cryostat/superconducting magnet system wired with cryogenic sub-miniature coaxial cable. Isothermal transfer curves were measured DC, with a voltage sweep rate of $50 \mathrm{mV} \mathrm{s}^{-1}$. Most of the temperature-dependent resistivity measurements were also performed DC, using a source/measure unit to source current, and a high-input-impedance, high-sensitivity multimeter to measure voltage. To improve signal-to-noise ratio in Hall and magnetoresistance measurements, a $13.7 \mathrm{~Hz}$ AC excitation was employed (using an AC resistance bridge), in the cases where the two-terminal resistance was $\sim 10 \mathrm{k} \Omega$ or lower. In all cases, extensive checks were made for self-heating and Ohmic response, particularly at low temperatures. Additional information on the methods employed for the transport measurements and their analysis is provided in Supplementary Figs S2-S5.

\section{References}

1. Heeger, A. J., Sariciftci, N. S. \& Namdas, E. B. Semiconducting and Metallic Polymers (Oxford University Press, 2010).

2. Skotheim, T. A. (ed.) Handbook of Conducting Polymers (Dekker, 1986).

3. Heeger, A. J., Kivelson, S., Schrieffer, J. R. \& Su, W.-P. Solitons in conducting polymers. Rev. Mod. Phys. 60, 781-850 (1988)

4. Stafstrom, S. Electron localization and the transition from adiabatic to nonadiabatic charge transport in organic conductors. Chem. Soc. Rev 39, 2484-2499 (2010).

5. Salleo, A., Kline, R. J., DeLongchamp, D. M. \& Chabinyc, M. L. Microstructural characterization and charge transport in thin films of conjugated polymers. Adv. Mater. 22, 3812-3838 (2010).

6. DeLongchamp, D. M., Kline, R. J., Fischer, D. A., Richter, L. J. \& Toney, M. F. Molecular characterization of organic electronic films. Adv. Mater. 23, 319-337 (2011)

7. Wang, Z. H., Li, C., Scherr, E. M., MacDiarmid, A. G. \& Epstein, A. J. Three dimensionality of "metallic" states in conducting polymers: Polyaniline. Phys. Rev. Lett. 66, 1745-1748 (1991).

8. Zuppiroli, L., Bussac, M. N., Paschen, S., Chauvet, O. \& Forro, L. Hopping in disordered conducting polymers. Phys. Rev. B 50, 5196-5203 (1994).

9. Yoon, C. O. et al. Hopping transport in doped conducting polymers in the insulating regime near the metal-insulator boundary: polypyrrole, polyaniline and poly-alkylthiophenes. Synth. Met. 75, 229-239 (1995).

10. Epstein, A. J., Lee, W.-P. \& Prigodin, V. N. Low-dimensional variable range hopping in conducting polymers. Synth. Met. 117, 9-13 (2001).

11. Lee, K. et al. Metallic transport in polyaniline. Nature 441, 65-68 (2006).

12. Menon, R., Yoon, C. O., Moses, D., Heeger, A. J. \& Cao, Y. Transport in polyaniline near the critical regime of the metal-insulator transition. Phys. Rev. B 48, 17685-17694 (1993).

13. Yoon, C. O., Reghu, M., Moses, D. \& Heeger, A. J. Transport near the metalinsulator transition: polypyrrole doped with $\mathrm{PF}_{6}$. Phys. Rev. B 49, 10851-10863 (1994).

14. Podzorov, V., Menard, E., Rogers, J. A. \& Gershenson, M. E. Hall effect in the accumulation layers on the surface of organic semiconductors. Phys. Rev. Lett. 95, 226601 (2005)

15. Takeya, J. et al. In-crystal and surface charge transport of electric-field-induced carriers in organic single-crystal semiconductors. Phys. Rev. Lett. 98, 196804 (2007).

16. Ahn, C. H. et al. Electrostatic modification of novel materials. Rev. Mod. Phys. 78, 1185-1212 (2006).

17. Sirringhaus, H. et al. Two-dimensional charge transport in self-organized, high-mobility conjugated polymers. Nature 410, 685-688 (1999).
18. Cho, J. H. et al. Printable ion-gel gate dielectrics for low-voltage polymer thin-film transistors on plastic. Nat. Mater. 7, 900-906 (2010).

19. Lodge, T. P. A unique platform for materials design. Science 321, 50-51 (2008).

20. Lee, J., Panzer, M. J., He, Y., Lodge, T. P. \& Frisbie, C. D. Ion gel gated polymer thin-film transistors. J. Am. Chem. Soc. 129, 4532-4533 (2007).

21. Lee, J. et al. Ion gel-gated polymer thin-film transistors: operating mechanism and characterization of gate dielectric capacitance, switching speed, and stability. J. Phys. Chem. C 113, 8972-8981 (2009).

22. Shimotani, H. et al. Insulator-to-metal transition in $\mathrm{ZnO}$ by electric double layer gating. Appl. Phys. Lett. 91, 082106 (2007).

23. Ye, J. et al. Liquid-gated interface superconductivity on an atomically flat film. Nat. Mater. 9, 8125-8128 (2010).

24. Dhoot, A. S. et al. Large electric field effect in electrolyte-gated manganites. Phys. Rev. Lett. 102, 136402 (2009).

25. Yamada, Y. et al. Electrically induced ferromagnetism at room temperature in cobalt-doped titanium dioxide. Science 332, 1065-1067 (2011).

26. Paulsen, B. \& Frisbie, C. D. Dependence of conductivity on charge density and electrochemical potential in polymer semiconductors gated with ionic liquids. J. Phys. Chem. C 116, 3132-3141 (2012).

27. Kline, R. J., Mcgehee, M. D. \& Toney, M. F. Highly oriented crystals at the buried interface in polythiophene thin-film transistors. Nat. Mater. 5, 222-228 (2006).

28. Mott, N. F. Metal Insulator Transitions. 2nd edn (Taylor and Francis, 1990).

29. Shklovskii, B. I. \& Efros, A. L. Electronic Properties of Doped Semiconductors (Springer-Verlag, 1984)

30. Sheng, P., Abeles, B. \& Arie, Y. Hopping conductivity in granular metals. Phys. Rev. Lett. 31, 44-47 (1973).

31. Castner, T. G. Hopping conduction in the critical regime approaching the metal-insulator transition. Pollak, M. \& Shklovskii, B. I. (eds) Hopping Transport in Solids (Elsevier, North-Holland, 1990).

32. Zhang, Y., Dai, P., Levy, M. \& Sarachik, M. P. Observation of a crossover from Mott to Efros-Shklovskii variable range hopping in n-CdSe. In Fritzsche, H. \& Pollak, M. (eds) Hopping and Related Phenomena (World Scientific, 1990).

33. Chen, S. A. \& Liao, C. H. Conductivity relaxation and chain motions in conjugated conducting polymers: neutral poly(3-alkylthiophenes). Macromolecules 26, 2810-2816 (1993).

34. Long, V. C., Washburn, S., Chen, X. L. \& Jenekhe, S. Hall-effect study of an ion-bombarded polymer. J. Appl. Phys. 80, 4202-4204 (1996).

35. Braga, D., Ha, M. J., Xie, W. \& Frisbie, C. D. Ultralow contact resistance in electrolyte-gated organic thin film transistors. Appl. Phys. Lett. 97, 193311 (2010).

\section{Acknowledgements}

This work was supported primarily by the MRSEC programme of the NSF under DMR-0819885. C.D.F. also acknowledges support from DOE BES, under DESC0004200. B. Shklovskii is acknowledged for useful discussions.

\section{Author contributions}

C.D.F. and C.L. conceived the project. S.W., M.H. and M.M. synthesized the samples, performed the experiments and analysed the data, under the supervision of C.L. and C.D.F. C.L., S.W. and C.D.F. wrote the paper.

\section{Additional information}

Supplementary Information accompanies this paper at http://www.nature.com/ naturecommunications

Competing financial interests: The authors declare no competing financial interests

Reprints and permission information is available online at http://npg.nature.com/ reprintsandpermissions/

How to cite this article: Wang, S. et al. Hopping transport and the Hall effect near the insulator-metal transition in electrochemically gated poly(3-hexylthiophene) transistors Nat. Commun. 3:1210 doi: 10.1038/ncomms2213 (2012). 